\title{
Producción científica de profesores en Universidades Peruanas: motivaciones y percepciones
}

\section{Scientific production of teachers in Peruvian Universities: motivations and perceptions}

\author{
Autores \\ Israel Barrutia Barreto. Universidad Científica del Sur. Lima. Perú. \\ E-mail: isbarisc@gmail.com https://orcid.org/0000-0002-5728-0651 \\ Erika Raquel Acosta Roa. Innova Scientific SAC. Lima. Perú. \\ E-mail:eacosta@innovascientific.com https://orcid.org/0000-0003-4011-4259 \\ Tomás Darío Marín Velásquez. Innova Scientific SAC. Lima. Perú. \\ E-mail: tmarin@innovascientific.com https://orcid.org/0000-0002-3334-5895
}

Fecha de recibido: 2019-10-18

Fecha de aceptado para publicación: 2019-12-20

Fecha de publicación: 2019-12-30

\section{Resumen}

El Perú ocupó en el año 2018 el séptimo lugar en producción científica en Latinoamérica, según el ranking Scimago, subiendo apenas dos puestos desde el año 2008. Por lo anterior, el estudio se centra en conocer la producción científica de docentes peruanos, comparando dos universidades, la Universidad Cesar Vallejo y Pontificia Universidad Católica del Perú. La investigación fue no experimental, descriptiva y comparativa. Se aplicó un muestreo probabilístico. Se utilizaron datos del Directorio Nacional de Investigadores e Innovadores, así como un cuestionario para obtener la motivación y percepción de los docentes. Se evidencia que la producción científica se desarrolla de manera desigual, favoreciendo a la Pontificia Universidad Católica del Perú, que presenta mayor desarrollo. A la mayoría de los docentes los motivan las normativas de educación superior del país (48\%) y se percibe falta de apoyo económico y asesoría técnica para la producción científica.

Palabras clave: Artículos científicos; investigación; producción científica; publicación y motivación; universidades.

\begin{abstract}
In 2018, Peru occupied the seventh place in scientific production in Latin America, according to the Scimago ranking, rising only two places since 2008. Therefore, the study focuses on knowing the scientific production of Peruvian teachers, comparing two universities, Universidad Cesar Vallejo and Pontificia Universidad Católica del Perú. The research was non-experimental, descriptive and comparative. A probability sampling was applied. Data from the National Directory of Researchers and Innovators were used, as well as a questionnaire to obtain the motivation and perception of teachers. It is evident that scientific production is developed in an unequal manner, favoring the Pontificia Universidad Católica del Perú, which presents greater development. The majority of teachers are motivated by the country's higher education regulations (48\%) and there is a perceived lack of economic support and technical advice for scientific production.
\end{abstract}

Keywords: Scientific articles; research; scientific production; publication and motivation; universities. 


\section{Introducción}

El contexto académico debe girar su atención a la generación de conocimientos, vistos como procesos que aportan a la productividad, este proceso se traduce en beneficios para la universidad y por ende para la sociedad, y la principal fuente de este conocimiento se encuentra inmerso en la producción científica, la cual puede englobar varios aprendizajes de conocimientos científicos y tecnológicos, despertando en el alumnado interés y vocación científica, que desarrolla un pensamiento transformador y, ocasiona un mayor apoyo al sistema de educación superior, a la inversión pública y a la sociedad. Es decir, la cultura científica es también la base para ampliar capacidades endógenas de innovación e investigación y desarrollo experimental (I+D) en la sociedad (Fernández, Bello \& Massarani, 2016).

En consecuencia del panorama anteriormente descrito, se espera que el docente pueda formar un ciudadano apto para la sociedad del conocimiento, por lo cual debe desarrollar habilidades que le permitan afrontar este desafío, una situación en la que subyacen varias estructuras, en cuanto a pertinentes reformas en los pensum de estudio universitarios, ya que estos responden a paradigmas superados (Salazar-Gómez \& Tobón, 2018) a fin de transformarlos y adaptarlos a las necesidades de instrucción de los estudiantes.

En este sentido, en Perú se han puesto en marcha iniciativas que se encaminan al fomento de la producción científica, pero pese a los esfuerzos realizados, dichas políticas públicas no han sido totalmente eficientes y eficaces para promover la producción científica, en cuanto a las universidades que más se destacan por ocupar los primeros puestos en el ranking mundial gracias a sus características de excelencia en la formación docente, estudiantil y en el campo la investigación, se puede mencionar la Pontificia Universidad Católica del Perú (PUCP), la cual es la primera del país según la Superintendencia Nacional de Educación Superior Universitaria (SUNEDU), en otro lugar se encuentra la Universidad Cesar Vallejo (UCV), la cual representa una de las universidades con más estudiantes a nivel nacional, a pesar de encontrarse en el puesto número 33 de las universidades según SUNEDU, además está dentro de las universidades que han recibido licenciamiento institucional y poseer una base de datos disponible para su estudio.

En líneas generales la producción científica en Perú se encuentra ligada a la actividad de investigación universitaria y se mide a través de una serie de indicadores relacionados fundamentalmente con la publicación de investigaciones bajo estándares internacionales, por 
ejemplo según datos del informe de SUNEDU en el 2018, el número de documentos citables y citas han elevado su número pero aún se encuentran en uno de los niveles más bajos de los países de América Latina, el número de revistas académicas indexadas en Scopus entre 2010 y 2015 pasó de cuatro a siete, aunque fue un crecimiento, la cifra no es significativa con respecto a los países de la región.

Con respecto a la producción científica del país por tipo de universidades, para el año 2016, las universidades asociativas presentan una mayor cantidad de publicaciones en revistas indexadas con 4568 artículos, seguidas por las universidades públicas con 3635 artículos, mientras que las universidades privadas societarias tienen una producción bastante más reducida con 447 artículos (SUNEDU, 2018).

Así mismo, según la información obtenida por el I Censo Nacional de Investigación y Desarrollo (SUNEDU, 2018) sobre el estatus de la investigación en el país para el año 2016, la producción científica es representada en primer lugar por los artículos científicos, libros y otras publicaciones. Las universidades públicas han producido 895 artículos científicos, 709 de ellos en revistas científicas indexadas, lo que representa un 79\%, y 186 en revistas no indexadas, un aporte de $21 \%$. De igual manera, el registro muestra que las universidades privadas, han producido 1412 artículos científicos, que representa un 58\% más que las universidades públicas, de los cuales 988 fueron publicados en revistas indexadas (70\%) y 424 se publicaron en revistas no indexadas $(30 \%)$

A causa de lo expuesto anteriormente, es preciso estudiar los niveles de productividad científica procedente de la actividad de investigación de los docentes de las universidades Cesar Vallejo y Pontificia Universidad Católica del Perú, traducidos en asesorías de tesis de pregrado y postgrado, publicaciones en revistas indexadas, nivel académico de los docentes, participación en proyectos de fondos concursables, producción intelectual, derechos de autor y registro de patentes, con el fin de determinar como la productividad científica impacta en el nivel académico y la ubicación de las universidades en ranking nacionales e internacionales, y como se relaciona esto con el desarrollo de la nación, ya que la difusión de conocimientos es un factor que refuerza el desarrollo, ante los acelerados cambios que se presentan en la sociedad actual. 


\section{Metodología}

Para la recolección de la información, se investigó en la base de datos del Concejo Nacional de Ciencia Tecnología e Innovación del Perú (CONCYTEC) de donde se obtuvo un total de 3526 docentes de las dos universidades, de los cuales se excluyeron aquellos que se encontraban registrados como investigadores (170) y los que no poseían la información requerida (103), por lo que quedaron 3253 docentes, distribuidos en 2558 de la UCV y 695 de la PUCP. Al aplicar la ecuación para el cálculo de muestra para poblaciones finitas (Aguilar-Barojas, 2005) se obtuvo una muestra de 334 docente de la UCV y 248 docentes de la PUCP.

Se aplicó un cuestionario de forma general a toda la muestra, para obtener la opinión de los docentes sobre la producción científica, el cual constó de cuatro preguntas, la primera con siete opciones, para conocer las motivaciones para generar producción científica. La segunda pregunta constó de tres opciones, para conocer la percepción sobre las políticas institucionales. La tercera pregunta, con ocho opciones, con la que se buscó establecer la percepción respecto a las dificultades e impedimentos para producción científica y por último una cuarta pregunta con cuatro opciones, para conocer la opinión sobre posibles medidas a implementar para mejorar las condiciones de la producción científica en las universidades. La investigación se planteó como no experimental, con diseño descriptivo y comparativo. Los datos obtenidos de CONCYTEC, fueron: el nivel académico, los artículos científicos publicados, proyectos de fondos concursables, patentes, derechos de autor y asesorías de tesis.

\section{Resultados}

Al comparar el nivel académico de los docentes de ambas universidades, se observó que, en las dos universidades, los porcentajes son similares. La mayoría de los docentes tienen grado de Maestría (57,2\% UCV y 52,4\% PUCP), igualmente en los grados de Doctor y Licenciados, la UCV muestra una ligera ventaja con 29,2 y 9,8\% respectivamente, en comparación con la PUCP con 21,0 y 8,5\%. Sin embargo, en la PUCP se constató una mayor proporción de Ingenieros con 10,6\%, en comparación con los de la UCV con el 2,9\%. Igualmente, la PUCP superó a la UCV en cuanto al número de Especialistas y bachilleres (profesionales que culminaron su educación superior, sin sustentar tesis para obtener la licenciatura). 
Respecto a los artículos publicados, se observó una clara diferencia a favor de la PUCP, donde el $28 \%$ de los docentes tomados como muestra, poseen artículos publicados, lo que contrasta con apenas 5\% de los profesores de la UCV. Este factor puede estar influyendo de manera determinante en el posicionamiento de ambas universidades dentro del ranking del país, donde la PUCP se encuentra en el puesto uno y la UCV en el puesto 33, según SUNEDU (2018). Así mismo, en la producción de las universidades respecto a los proyectos presentados a fondos concursables, patentes y derecho de autor, se destaca la PUCP en relación a los proyectos de fondos concursables, observándose que la mayoría de los docentes muestreados, no poseen algún tipo de propiedad intelectual (98\% UCV y 92\% PUCP), sin embargo la UCV posee una mayor cantidad de docentes con derecho de autor (2\%) y en la PUCP se encuentran más docentes con patentes $(2 \%)$ y proyectos de fondos concursables $(6 \%)$.

También se observó que la mayoría de los docentes, no posee asesorías de tesis en ningún nivel (62\% UCV y 66\% PUCP). En cuanto a las asesorías en pregrado, un mayor porcentaje de los docentes de la UCV poseen asesorías (24\%), comparados con lo obtenido para los docentes de la PUCP (18\%). En relación con las asesorías de postgrado (Maestría y Doctorado) en ambas universidades se observan porcentajes similares, encontrándose que, de los docentes de la PUCP, el 12\% y 4\% asesoran tesis de Maestría y Doctorado, mientras que en la UCV los porcentajes son de $11 \%$ y $3 \%$.

La primera pregunta, se redactó de la siguiente manera: ¿Cuáles considera que son las razones para generar producción científica? Para la mayoría de los docentes, la principal motivación para la producción científica es, por requerimientos de la educación superior del país (48\%), lo cual se debe a la implementación de normativas que exigen a los docentes poseer producción científica, para poder ser clasificados y mantenerse como docentes-investigadores. Lo que incide en la segunda opción con mayor porcentaje, que es la motivación laboral y económica (24\%), debido a que existe una relación directa entre estas dos opciones, pues las normas implementadas conllevan mejoras tanto laborales como económicas. Otro porcentaje importante (15\%) considera la mejora del currículum como motivación para la generación de producción científica. En contraste se observa que la difusión de la ciencia y la solución de problemas nacionales son las menores motivaciones para la producción científica, por lo que sólo 3 y $1 \%$ de los docentes en general las señalan como motivación. 
Los resultados de la segunda pregunta, la cual se redactó como: ¿Considera que existen políticas para incentivar la producción científica en la institución?, mostró que predomina la percepción negativa en cada una de las opciones. Los docentes encuestados evalúan negativamente las políticas de las instituciones, respecto a incentivar la producción científica. Se observa que la percepción es que las instituciones en general no cuentan con políticas para la publicación, ni recursos, ni políticas de formación de los docentes $(58,75$ y 69\%) respectivamente. Las apreciaciones positivas se inclinaron hacia las políticas para publicación (42\%), influenciadas por las directrices emanadas de CONCYTEC y de SUNEDU, ya que una parte importante de los requisitos, para el licenciamiento institucional de las universidades recae en la investigación y publicación científica.

Para la tercera presunta: ¿Cuáles considera que son las principales dificultades para la producción científica?, se obtuvo que el aspecto económico es el predominante. La percepción general de los profesores respecto a las dificultades para la producción científica, se centra principalmente en la falta de apoyo económico que incentive a los docentes a investigar y producir proyectos, artículos y patentes (46\%). Es evidente que los resultados de esta pregunta se relacionan con lo observado en las anteriores, donde el aspecto económico se presenta como el más representativo, según la opinión general. La falta de asesoría y de capacitación técnica con 21 y 15\%, son otras opciones importantes, que además concuerdan con la percepción negativa observada en la tercera pregunta. Como las opciones menos relevantes, se muestran la falta de resultados de investigación, la falta de espacios adecuados y la falta de bibliografía, de lo que se deduce que los docentes consideran que si se cuenta con resultados de investigación acordes para ser publicados en revistas científicas, también existe la infraestructura necesaria para investigar en las universidades, así como la falta de bibliografía no es relevante ya que las universidades ponen a disposición de los docentes, bases bibliográficas y repositorios.

La cuarta pregunta, se redactó para conocer la opinión de los docentes sobre las medidas de mejora: ¿Desde su punto de vista, ¿cuáles serían las posibles medidas para mejorar el nivel de producción científica? Los resultados se muestran, al igual que se observó en las preguntas anteriores, un sesgo hacia el aspecto económico. Como se mencionó, los profesores consideraron que la medida más relevante a tomar en cuenta para incentivar la producción científica de los docentes, es la asignación de mayor cantidad de recursos económicos (40\%). Así mismo, el aspecto formativo con la promoción de cursos, talleres y asesorías, se muestra 
como otra opción de importancia con $37 \%$, el apoyo institucional a la investigación y el fortalecimiento de las revistas científicas propias, fueron las opciones con menos porcentaje con 14 y $9 \%$ respectivamente.

\section{Discusión}

En las dos universidades tomadas como caso de estudio, la mayoría de los docentes poseen grados académicos de Magíster y Doctor (86,4 UCV y 73,4\% PUCP) lo que contrasta con la baja producción científica, esto si es tomado en cuenta que, un grado de Maestría académica o Doctorado, se refiere a "estudios de carácter académico basados en la investigación” según la Ley universitaria No 30220 de la República del Perú del año 2014 (Ley N³0220, 2014). La citada ley también especifica que un profesional con grado académico de Doctor debe tener además de las competencias investigativas, “conocimientos del más alto nivel”. En opinión de Guerrero (2011), es claro que la formación para la investigación debe hacer parte de los programas académicos de Maestría y de Doctorado, que permitan generar competencias que sirvan de punto de partida para la creación e innovación. Por otro lado, los porcentajes de profesores con grados académicos de cuarto y quinto nivel es acorde con lo que establece la Ley No 30220 de Perú, donde se exige esos grados para ejercer la docencia universitaria.

La producción científica se muestra baja en ambas universidades, lo que es más notorio en la UCV donde sólo el 5\% de los docentes posee publicaciones en revistas indexadas, a pesar de contar con mayor número de docentes con grados académicos de Magíster y Doctorado. La producción de artículos científicos en el país es en líneas generales bajo, por lo que las actuales normativas de CONCYTEC buscan aumentar las cifras, promulgando nuevos estándares para la clasificación de docentes investigadores, lo que evidentemente va a influir en el aumento de la producción científica. La proporción de docentes que publican artículos científicos en la PUCP es superior (28\%) lo que evidentemente influye en que la misma esté en la primera posición en el ranking de las universidades peruanas (SUNEDU, 2018).

En relación con otras producciones científicas, como los proyectos de fondos concursables, las patentes y los derechos de autor, la cantidad de docentes que las poseen es mucho menor, en comparación con los artículos científicos, llegando a ser en la UCV de solo 2\% y en la PUCP de $8 \%$. Se puede decir que uno de los factores más influyentes en estos datos estadísticos es que la mayoría de los profesores de las dos universidades tienen estatus laboral de contratados, que se agrava por ser instituciones de carácter privado, como lo indica el portal del Vicerrectorado 
Académico de la PUCP en octubre de 2018, donde se indica que el 78\% de los docentes universitarios, en estas casas de estudios superiores, son contratados. Al no ser la mayoría profesores nombrados, existe poco sentido de pertenencia, como lo indica Valcazar (2019), lo que evidentemente influye en el compromiso de los mismos con las instituciones y por ende con la investigación y la producción científica. El porcentaje de docentes con asesorías de tesis, también se muestra bajo en las dos universidades (38\% UCV y 34\% PUCP) si se toma en cuenta el grado académico de los profesores.

La producción científica, como se ha observado, está mayoritariamente ligada a la publicación de artículos y a las asesorías de tesis, esto también fue observado por Codina-Canet, OlmedaGómez y Perianes-Rodríguez (2013) quienes al analizar la producción científica de la Universidad Politécnica de Valencia durante los años 2003-2010, concluyen que un tercio de los trabajos se publican en forma de comunicaciones a congresos y los publicados en forma de artículos representan el 95\% de la producción científica. Así mismo, Castillo y Powell (2019) también muestran que, para el caso específico de Ecuador, el 75\% de la producción científica entre el 2006 y el 2015 fueron artículos científicos, lo que es consistente con lo observado en la presente investigación.

Al analizar la motivación de los docentes para generar producción científica, se observa que la mayoría se inclina por las normativas de la educación superior del Perú (48\%), lo que evidentemente está ligado al beneficio económico, por lo que se observa que el 24\% también lo mencionan como motivo. Los resultados contrastan con los mostrados por Pinto-Santos, Villanueva-Valadez y Cortés-Peña (2019) quienes obtuvieron que en las universidades Uniguajira de Colombia y la Universidad de Nuevo León de México, la principal motivación fue la visualización de las investigaciones con 44,6 y 47,1\% respectivamente, sin embargo, los autores citados también destacan que en la Universidad de Nuevo León un 28,6\% de los docentes consideran las exigencias laborales como motivación.

Los factores que influyen en la baja producción científica, de acuerdo a los docentes encuestados, se concentran en la falta de apoyo económico y la falta de asesoría y de capacitación técnica, lo que es un resultado contrastante con el obtenido por Escobar-Pérez, García-Meca y Larrán-Jorge (2014) quienes al aplicar encuestas a docentes de varias universidades españolas para conocer la percepción sobre los factores que influyen sobre la producción científica en Contabilidad, obtuvieron que los profesores se inclinan, a nivel global 
por la desigualdad de criterios entre las áreas del conocimiento y la dificultad para diferenciar entre la investigación y los trabajos orientados a la mejora de la práctica profesional como uno de los principales factores que inciden en la producción científica, así como también, la desmotivación por los rechazos sufridos y la presencia de actitudes acomodadas, seguidas por la falta de incentivos a la investigación, tanto en forma de financiación, como de reconocimiento al asignar la docencia. De igual forma, lo obtenido por Rueda-Barrios y Rodenes-Adam (2016) al analizar la producción de grupos científicos de Colombia, mostró que los factores institucionales asociados a la producción científica, tales como el tiempo de dedicación para la investigación, disponibilidad de recursos, y visibilidad de los resultados de investigación, fueron determinantes en la generación de la producción.

El mayor porcentaje de los docentes manifiesta que se debe incrementar el apoyo económico a la producción científica, así como también generar un proceso formativo que conlleve a incentivar la investigación y la producción científica, lo que está en línea con lo manifestado por Martelo, Jaramillo y Ospino (2018) quienes al evaluar la producción científica de docentes universitarios, concluyeron que los aspectos que deben ser tomados en cuenta y mejorados, son el reconocimiento a los docentes, capacitación, apoyo para grupos nuevos de investigación y disponibilidad de recursos de investigación.

\section{Conclusiones}

Al explorar la productividad de los docentes universitarios de las universidades Cesar Vallejo y la Pontificia Universidad Católica de Perú, se puede constatar que la producción científica presenta una proporción desigual en el desarrollo de las actividades de investigación, lo cual se refleja en los resultados de la producción científica intelectual, la publicación de artículos en revistas indexadas.

Se concluye que la producción científica de los docentes de ambas universidades es incipiente, lo cual afecta el entorno educativo, ya que el docente no se está haciendo participe de una de las fases primordiales del proceso de enseñanza y educación como lo es el área de la investigación, lo que ocasiona que no se estén generando nuevas investigaciones o trabajos innovadores que repercutan de manera positiva en el desarrollo del país.

Se puede decir que la situación está relacionada, según la apreciación de los docentes, con elementos como: la falta de políticas por parte de las autoridades universitarias, el irrisorio 
presupuesto asignado por el Estado para fortalecer la producción científica en las instituciones de educación superior, la poca o nula remuneración asignada a los docentes que realizan trabajos de investigación y la falta de formación de los docentes en investigación, lo que origina que la gran mayoría de ellos abandone el rol de investigador y se cargue de horas académicas a fin de elevar su remuneración mensual.

En tal sentido, se recomienda diseñar programas que incentiven e integren a los docentes a través de acciones que satisfagan las necesidades antes mencionadas, y difundir políticas de Estado dirigidas al fomento de la producción científica en todas las modalidades de la educación superior, que integren al docente como parte primordial del proceso de producción científica con el propósito de formar una generación de relevo que posea las herramientas pertinentes que le hagan un profesional investigador.

\section{Referencias}

Aguilar-Barojas, S. (2005). Fórmulas para el cálculo de la muestra en investigaciones de salud. Salud en Tabasco, 11(2), 333-338.

Castillo, J.A. \& Powell, M.A. (2019). Análisis de la producción científica del Ecuador e impacto de la colaboración internacional en el periodo 2006-2015.Revista Española de Documentación Científica, 42(1), 1-16. doi: 10.3989/redc.2019.1.1567

Codina-Canet, M.A., Olmeda-Gómez, C. \& Perianes-Rodríguez, A. (2013). Análisis de la producción científica y de la especialización temática de la Universidad Politécnica de Valencia. Scopus (2003-2010). Revista Española de Documentación Científica, 36(3), 1-17. doi: 10.3989/redc.2013.3.942

Decreto 1001/2006. Reglamento de programas de posgrado de Colombia. Bogotá: Ministerio de Educación Nacional.

Escobar-Pérez, B., García-Meca, E. \& Larrán-Jorge, M. (2014). Factores que influyen sobre la producción científica en Contabilidad en España: la opinión de los profesores universitarios de Contabilidad (II parte). Revista Española de Documentación Científica, 37(2), 1-15. doi: 10.3989/redc.2014.2.1087

Fernández, E., Bello, A. \& Massarani, L. (2016). Políticas públicas e instrumentos para el desarrollo de la cultura científica en América Latina. Montevideo: UNESCO 
Guerrero, M.E. (2011). Formación para la investigación y programas de posgrado. Studiositas. 6(1), 19-35.

Ley N $\mathrm{N}^{\mathrm{a}}$ 30220/ 2014, de 09 de julio, Ley universitaria del Perú. Lima: Ministerio de Educación.

Martelo, R., Jaramillo, J. \& Ospino, M. (2018). Producción científica de docentes universitarios y estrategias para aumentarla mediante series de tiempo y MULTIPOL. Espacios, 39(16), 11-21.

Pinto-Santos, A., Villanueva-Valadez, C. \& Cortés-Peña, O. (2019). Percepciones del docente universitario sobre la comunicación científica de libre acceso. Revista Española de Documentación Científica, 42(2), 1-15. doi: 10.3989/redc.2019.2.1588

Rueda-Barrios, G. \& Rodenes-Adam, M. (2016). Factores determinantes en la producción científica de los grupos de investigación en Colombia. Revista Española de Documentación Científica, 39(1), 1-16. doi: 10.3989/redc.2016.1.1198

Salazar-Gómez, E. \& Tobón, S. (2018). Análisis documental del proceso de formación docente acorde con la sociedad del conocimiento. Espacios, 35(53), 17-30.

SUNEDU. (2018). Informe bienal sobre la realidad universitaria peruana. Recuperado de: https://www.sunedu.gob.pe/informe-bienal-sobre-realidad-universitaria/

Valcazar, E. (2019). Las competencias del docente de posgrado. Un estudio comparativo en cuatro maestrías especializadas desde la percepción de los estudiantes. Desde el Sur, 11(1), 191-206. doi: 10.21142/DES-1101-2019-191-206

Vicerrectorado Académico PUCP. (2018). Incertidumbre y desigualdad: La situación de los profesores contratados. Recuperado de:

http://vicerrectorado.pucp.edu.pe/academico/noticias/incertidumbre-desigualdadprofesores-contratados/ 